\title{
El trabajo sociocultural comunitario: misión de la educación superior
}

Dianelkys Martínez-Rodríguez

\section{RESUMEN}

Elevar los niveles de impacto de la educación superior en el orden social constituye una prioridad del nuevo milenio, para el cumplimiento de los Objetivos de Desarrollo Sostenible. Cada día se hace más necesaria la sinergia entre los procesos de formación, investigación y extensión universitaria, para la transformación de realidades socioculturales insostenibles. Este artículo tiene dentro de sus propósitos, analizar los aportes teórico-metodológicos que sustentan la misión educativa de la educación superior para el desarrollo sostenible. En la fase exploratoria, la revisión de la literatura se realizó a partir de las categorías Vínculo universidad-sociedad y Vínculo universidad-comunidad. Posteriormente se utilizaron los criterios de la Teoría fundamentada para profundizar en los principales hallazgos teóricos. En los análisis se defiende el término trabajo sociocultural comunitario y se presentan cuatro principios que permitan reflexionar sobre el salto cualitativo que requieren las prácticas educativas a este nivel para 2030.

Palabras clave: educación superior, sociedad, desarrollo sustentable, cultura, desarrollo de la comunidad. 


\title{
RESUMEN
}

Elevar los niveles de impacto de la educación superior en el orden social constituye una prioridad del nuevo milenio, para el cumplimiento de los Objetivos de Desarrollo Sostenible. Cada día se hace más necesaria la sinergia entre los procesos de formación, investigación y extensión universitaria, para la transformación de realidades socioculturales insostenibles. Este artículo tiene dentro de sus propósitos, analizar los aportes teórico-metodológicos que sustentan la misión educativa de la educación superior para el desarrollo sostenible. En la fase exploratoria, la revisión de la literatura se realizó a partir de las categorías Vínculo universidad-sociedad y Vínculo universidad-comunidad. Posteriormente se utilizaron los criterios de la Teoría fundamentada para profundizar en los principales hallazgos teóricos. En los análisis se defiende el término trabajo sociocultural comunitario y se presentan cuatro principios que permitan reflexionar sobre el salto cualitativo que requieren las prácticas educativas a este nivel para 2030.

Palabras clave: educación superior, sociedad, desarrollo sustentable, cultura, desarrollo de la comunidad.

\section{O trabalho sociocultural comunitário: missão do ensino superior}

\section{RESUMO}

Elevar os níveis de impacto do ensino superior na ordem social constitui uma prioridade do novo milênio, para cumprir os Objetivos de Desenvolvimento Sustentável. Cada dia se faz mais necessária a sinergia entre os processos de formação, pesquisa e extensão universitária, para a transformação de realidades socioculturais insustáveis. Este artigo tem dentro de seus propósitos analisar as contribuições teórico-metodológicas que sustentam a missão educativa do ensino superior para o desenvolvimento sustentável. Na fase exploratória, a revisão da literatura se realizou a partir das categorias: Vínculo Universidade-Sociedade e Vínculo Universidade-Comunidade. Posteriormente se utilizaram os critérios da Teoria fundamentada para aprofundar nas principais descobertas teóricas. Nas análises se defende o termo trabalho sociocultural comunitário e se apresentam quatro princípios que permitam refletir sobre o salto qualitativo que requerem as práticas educativas a este nível para 2030.

Palavras chave: educação superior, sociedade, desenvolvimento sustentável, cultura, desenvolvimento da comunidade.

\section{Community Socio-cultural Work: The Mission of Higher Education}

\begin{abstract}
Raising the impact of higher education on the social order is a priority of the new millennium for the achievement of the Sustainable Development Goals. Synergy between the processes of training, research and university extension is becoming increasingly necessary for the transformation of unsustainable socio-cultural realities. One of the purposes of this article is to analyze the theoretical-methodological contributions that support the educational mission of higher education for sustainable development. In the exploratory phase, the literature review was carried out from the categories: University-Society Link and University-Community Link. Subsequently, the criteria of Grounded Theory were used to deepen the main theoretical findings. In the analysis, the term community socio-cultural work is defended and four principles are presented that allow us to reflect on the qualitative leap required by educational practices at this level by 2030 .
\end{abstract}

Key words: higher education, society, sustainable development, culture, community development. 


\section{Introducción}

La misión educativa de la educación superior de acuerdo con los 17 objetivos de desarrollo sostenible (ODS) contenidos en la Agenda 2030 para el Desarrollo Sostenible, requiere ser perfeccionada para elevar su pertinencia. Aun cuando los debates internacionales han dado muestra de esta preocupación desde 1998, los documentos generados que sustentan los marcos de acción global y local, demandan acciones concretas que incorporen modelos, metodologías, concepciones o estrategias educativas, pedagógicas y/o didácticas.

$\mathrm{El}$ interés por identificar esos aportes teóricos y metodológicos, que hagan pertinente la misión educativa de la educación superior para el desarrollo sostenible, fue la guía fundamental de la fase exploratoria de esta investigación, en la cual la detección de la literatura se ubicó en dos vínculos fundamentales: universidad-sociedad y universidad-comunidad. Estas relaciones surgen de analizar los antecedentes y evolución de los pronunciamientos internacionales acerca de la misión educativa de la educación superior, de los cuales se presentan a continuación algunas ideas esenciales.

En la antesala del siglo XXI, en la Conferencia Mundial sobre la Educación Superior de la Organización de Naciones Unidas para la Educación, la Ciencia y la Cultura (UNESCO) realizada en 1998, Federico Mayor habló de la importancia de "las misiones de la educación superior, su apertura a toda la sociedad y su interacción con los principales actores sociales y con los grandes sectores" (UNESCO, 1998: 4). En los análisis realizados en dicho espacio se expresó que "la pertinencia de la educación superior debe evaluarse en función de la adecuación entre lo que la sociedad espera de las instituciones y lo que éstas hacen" (ibid.: 2).

En los criterios de la UNESCO se aprecia que la misión educativa de la educación superior es la atención a las problemáticas sociales que en un orden mundial requieren de una actuación contextualizada, sustentada en los aportes de la actividad científico-investigativa y de la innovación, así como la interrelación de éstas con los restantes procesos sustantivos (la docencia y la extensión universitaria). Se trata de educar para una profesión y para la vida, desde una concepción que conduzca a la reflexión, autorreflexión y acción transformadora.

La educación superior debe atender problemas tales como: "la pobreza, la intolerancia, la violencia, el analfabetismo, el hambre, el deterioro del medio ambiente y las enfermedades, y a las actividades encaminadas al fomento de la paz, mediante un planteamiento interdisciplinario y transdisciplinario" (UNESCO, 1998: 2).

La importancia de la misión educativa de la educación superior, "consiste en favorecer el desarrollo integral de la persona y formar ciudadanos responsables, informados, comprometidos para actuar en pro de un futuro mejor para la sociedad" (UNESCO, 1998: 4).

En la Conferencia Mundial de la Educación Superior celebrada por la UNESCO en 2009, las ideas anteriores se enfatizan y se desarrollan bajo el concepto de la responsabilidad social, término mediante el cual se expresa la misión educativa de la educación superior, presente en aquellas instituciones donde se asume el liderazgo social en materia de creación de conocimientos de alcance mundial, para abordar retos mundiales y locales, entre los que figuran la seguridad alimentaria, el cambio climático, la gestión del agua, el diálogo intercultural, las energías renovables y la salud pública.

La Reunión Mundial sobre la Educación para Todos (UNESCO, 2014a), la Conferencia Mundial para el Desarrollo Sostenible (UNESCO, 2014b), la Declaración de Incheon del Foro Mundial sobre la Educación (UNESCO, 2015), el Documento de posición sobre la Educación después de 2015 (UNESCO, 2015), han evidenciado que la educación, en sentido general, representa un eje transversal para el desarrollo sostenible, condición que igualmente corresponde a la educación superior y su proyección hacia una constante revolución educativa en su interior y para las comunidades externas. 
La Organización de Naciones Unidas (ONU), refuerza en la Agenda 2030 las pautas establecidas por la UNESCO sobre la concepción integral de la "Educación para Todos" y durante toda la vida, lo que también implica a su nivel superior. Dentro de los ODS se plantea la necesidad de:

\begin{abstract}
asegurar que todos los alumnos adquieran los conocimientos teóricos y prácticos necesarios para promover el desarrollo sostenible, entre otras cosas mediante la educación para el desarrollo sostenible y los estilos de vida sostenibles, los derechos humanos, la igualdad de género, la promoción de una cultura de paz y no violencia, la ciudadanía mundial y la valoración de la diversidad cultural y la contribución de la cultura al desarrollo sostenible (ONU, 2015: 20).
\end{abstract}

En los ODS se identifican ejes transversales para la formación de pregrado, acordes con el papel que debe jugar la educación superior en el nuevo milenio. No es suficiente con la enseñanza de las materias tradicionales presentes en el currículo de las carreras, sino que se trata de determinar cuáles serán los conocimientos, habilidades y valores acordes con la participación social en la solución de problemas desde el principio de la sostenibilidad.

En correspondencia con la idea anterior, en el $10 \mathrm{mo}$ Congreso Internacional de Educación Superior celebrado en La Habana, se reflexiona en torno al concepto de universidad innovadora: "antítesis de organizaciones estáticas, sujetas a modelos que pertenecen al pasado. Es la universidad que se reforma permanentemente, enriqueciendo su modelo de gestión, para cumplir mejor su función social mediante la sinergia de las actividades de formación, investigación y la extensión universitarias, vinculadas siempre con la sociedad" (Alarcón, 2016: 8).

Este concepto de universidad innovadora es expresión de la constante transferencia del conocimiento que debe producirse y gestionarse para la sociedad, a través del desarrollo sinérgico de los procesos sustantivos de la educación superior. Forma parte del discurso en el "deber ser" de las prácticas institucionales actuales.

Ulterior a la etapa de revisión de la literatura, la investigación se orientó a los siguientes propósitos: 1. Analizar los aportes teóricos y metodológicos publicados en revistas científicas, que contuvieran propuestas significativas al perfeccionamiento de la misión educativa de la educación superior para el desarrollo sostenible; 2. Identificar aportes teóricos y metodológicos emergentes del análisis e interpretación de los datos, que puedan contribuir al perfeccionamiento de la misión educativa de la educación superior para el desarrollo sostenible.

\section{El proceso de recopilación y análisis de los datos}

Para la recopilación de los datos se utilizaron buscadores digitales especializados en educación (EBSCO, ERIC, Dialnet, Google académico y Redalyc), el tesauro UNESCO y el Vocabulario controlado de IRESIE, esencialmente. La consulta de los artículos científicos se realizó bajo los siguientes criterios: a) fecha de publicación, comprendida entre 2011 y 2016, b) contexto donde se realizó la investigación, c) validación empírica de los aportes teóricos y metodológicos a partir de las categorías de análisis en un plano inicial (categoría 1, vinculación universidadsociedad y categoría 2, vinculación universidadcomunidad). El análisis de los datos se realizó desde un enfoque cualitativo y siguiendo los criterios de la Teoría fundamentada, "lo cual significa que la teoría (hallazgos) va emergiendo fundamentada en los datos" (Hernández-Sampieri et al., 2010: 444).

Para la interpretación de los datos se consideraron: a) las definiciones y descripciones teórico-conceptuales presentes en el vínculo universidad-sociedad-comunidad, b) las relaciones entre categorías del plano inicial y las categorías emergentes en el análisis de los datos (cuadro 1), c) la frecuencia con que son utilizadas las categorías del plano inicial y las categorías emergentes. 


\section{Cuadro 1. Categorías analizadas en el estudio}

\begin{tabular}{|c|c|l|}
\hline Categorías del plano inicial & Categorías emergentes & \multicolumn{1}{c|}{ Subcategorías emergentes } \\
\hline \multirow{2}{*}{ Universidad-sociedad-comunidad } & - Trabajo comunitario. & - Servicio comunitario. \\
& - Cooperación sociocultural. & - Aprendizaje-servicio. \\
& - Extensión universitaria. & • Vinculación comunitaria. \\
\hline
\end{tabular}

Fuente: elaboración propia.

\section{Experiencias teórico-metodológicas de una educación superior para el desarrollo sostenible}

Las universidades están reconocidas como las instituciones donde se concreta la misión educativa de la educación superior. Se les considera un móvil potencial para el crecimiento económico, un espacio para la creación e innovación y la aplicación del conocimiento teórico, dirigido a los desafíos medioambientales, con un impacto significativo (positivo o negativo) en la distribución de la riqueza de una sociedad; además, potencialmente mantienen un espacio para la discusión de modelos de desarrollo local, nacional y global (Mc Cowan, 2016).

Las experiencias teórico-metodológicas que contribuyen a la educación superior para el desarrollo sostenible, provienen de prácticas diversas en universidades del contexto iberoamericano, las cuales han estado centradas en el trabajo comunitario (Pérez, 2011; Ruiz, 2011; García, 2013; Gregorutti y Charles-Marcel, 2013; Sánchez y Caldera, 2013; Ávila et al., 2016; Castro et al., 2016); la animación sociocultural (Pérez-Pérez, 2014); la cooperación interuniversitaria (Melero y Fleitas, 2015), y la extensión universitaria como experiencia formativa estudiantil (Restrepo-Salazar et al., 2015) (cuadro 2).

El trabajo comunitario es la experiencia teóricometodológica de mayor presencia en la misión educativa de las universidades, que se asume como un servicio desde diferentes criterios: mecanismo de vinculación de las universidades con su entorno (Pérez, 2011); vía o herramienta de la enseñanza y el aprendizaje (Ruiz, 2011); modelo de intervención (Gregorutti y Charles-Marcel, 2013) y modelo educativo (Ávila et al., 2016).

El servicio comunitario de estudiantes "representa gran oportunidad de hacer de las universidades, centros de investigación socialmente responsables, y es un excelente mecanismo de vinculación de las universidades con su entorno, sensibilizando a todos los integrantes que la componen" (Pérez, 2011 : 204).

Para Pérez (2011), el servicio comunitario de los estudiantes es una estrategia de formación de pregrado a partir de la participación de los protagonistas académicos con los protagonistas comunitarios; sin embargo, aun cuando propicia un proceso de enseñanza y aprendizaje vinculado a la realidad social, en espacios de un nivel micro, la experiencia en la práctica educativa ha demostrado que son débiles los vínculos con sectores públicos, privados y gubernamentales, así como el enfoque con que se asume esta responsabilidad social universitaria (RSU), "vista sólo como cultura y arte" (Pérez, 2011: 206).

$\mathrm{El}$ aprendizaje-servicio, aunque se valora como "herramienta valiosa en todas aquellas actividades que abarcan, al mismo tiempo, objetivos de intervención comunitaria y de aprendizaje" (Ruiz, 2011: 254-255); se muestra como una estrategia educativa centrada en la vinculación del currículo tradicional, con el currículo emergente del intercambio con la comunidad: 


\section{Cuadro 2. Experiencias de la relación Universidad-Sociedad-Comunidad}

\begin{tabular}{|c|c|c|c|}
\hline Autor/año & $\begin{array}{l}\text { Tipo de } \\
\text { estudio }\end{array}$ & Experiencias & Aportes teórico-metodológicos \\
\hline Pérez/2011 & Teórico & Proyecto de servicio comunitario. & $\begin{array}{l}\text { Responsabilidad social universitaria (RSU). } \\
\text { Transferencia de conocimientos. }\end{array}$ \\
\hline Ruiz/2011 & Empírico & $\begin{array}{l}\text { Concepto de aprendizaje- } \\
\text { servicio. }\end{array}$ & Educación integral. \\
\hline García/2013 & Teórico & $\begin{array}{l}\text { Articulación entre universidad- } \\
\text { escuela-comunidad. }\end{array}$ & $\begin{array}{l}\text { Investigación-acción participativa (IAP). } \\
\text { Concepción de la Educación problematizadora y liberadora. }\end{array}$ \\
\hline Gregorutti y Charles-Marcel/2013 & Empírico & $\begin{array}{l}\text { Servicio comunitario integrado a } \\
\text { la enseñanza. }\end{array}$ & $\begin{array}{l}\text { Promoción de estilos de vida saludable como parte del trabajo } \\
\text { comunitario. } \\
\text { Metodología de inclusión de actores sociales. }\end{array}$ \\
\hline Sánchez y Caldera/2013 & Teórico & Proyectos comunitarios. & $\begin{array}{l}\text { Teoría del trabajo comunitario (participación y planificación, } \\
\text { liderazgo). } \\
\text { Responsabilidad social. }\end{array}$ \\
\hline Pérez/2014 & Empírico & $\begin{array}{l}\text { Interdisciplinariedad en } \\
\text { el proceso de enseñanza- } \\
\text { aprendizaje. }\end{array}$ & $\begin{array}{l}\text { Educación para el desarrollo. } \\
\text { Animación sociocultural. } \\
\text { Desarrollo comunitario. } \\
\text { Participación. Pedagogía social y educación social. }\end{array}$ \\
\hline Melero y Fleitas/2015 & Empírico & Cooperación interuniversitaria. & IAP. \\
\hline Restrepo et al./2015 & Teórico & $\begin{array}{l}\text { Incorporación de la extensión } \\
\text { universitaria al currículo. }\end{array}$ & RSU. \\
\hline Ávila et al./2016 & Empírico & $\begin{array}{l}\text { Experiencias de educación } \\
\text { superior intercultural. }\end{array}$ & Teoría de la dialogicidad de Paulo Freire. \\
\hline Castro et al. 2016 & Teórico & $\begin{array}{l}\text { Vinculación comunitaria y } \\
\text { formación académica. }\end{array}$ & Dialogicidad e interdisciplinariedad. \\
\hline
\end{tabular}




\begin{abstract}
Se puede hablar de aprendizaje-servicio cuando es planificado no sólo en función de las demandas de la comunidad sino en función del proyecto educativo institucional, el cual tiene por finalidad desarrollar una acción de carácter solidario relacionada con la promoción de valores y actitudes en donde exista una articulación de la actividad que se realiza en la comunidad con los contenidos curriculares que se llevan a la práctica en el aula (Ruiz, 2011: 254).
\end{abstract}

Pérez (2011) y Ruiz (2011), analizan esta relación universidad-comunidad desde los vínculos entre la Ley de Servicio Comunitario del Estudiante de Educación Superior (LSCEES) existente en Venezuela, y la experiencia valorativa-práctica en carreras tanto de corte social o humanista, como técnico. En ambos casos se aprecian como aspectos positivos: 1) el interés y sensibilización del sistema de educación superior, estudiantes y profesores, por estrechar las distancia entre la academia y su contexto; 2) el reconocimiento del valor que tiene el trabajo comunitario en la formación de futuros profesionales. No obstante, resultan insuficientes: a) la transformación pedagógica y didáctica de la universidad para alcanzar el aprendizaje-servicio; b) la implicación de diversos actores sociales e institucionales; c) el equilibrio de los intereses educativos con las necesidades sentidas de la comunidad, y d) la credibilidad en la intervención comunitaria por parte de las comunidades.

Desde el punto de vista didáctico, García (2013: 203) defiende la idea de un "curriculum contrahegemónico, que incorpore los problemas sociales, económicos, políticos, etcétera, que permita tomar conciencia de la realidad para actuar sobre ella con el fin de contribuir a la transformación del modelo social existente".

La propuesta teórica de García, se fundamenta en el paradigma sociocrítico de la investigación social y cultural, mediante el cual propone una metodología pedagógica basada en proyectos de aprendizaje servicio y de investigación-acción-crítica-participativa, lo que permita vincular "los contenidos de las diferentes asignaturas académicas a problemas y necesidades detectadas y manifestadas en las comunidades, instituciones o instancias sociales y educativas, actuando a partir de éstas para promover cambios con y desde las comunidades correspondientes" (García, 2013: 203).

Así, la concepción pedagógica de Freire está presente en la propuesta de García (2013) y eso la hace valiosa, pues en ella se reconoce la necesidad de problematizar sobre la realidad, contextualizar lo que se enseña y la participación de educandos y educadores en un proceso colectivo, cooperativo y solidario.

Desde las teorías en que García asume su propuesta, se considera que la comunidad se convierte en una protagonista importante en la formación de los estudiantes universitarios. La unidad de saberes (académicos y populares) es una muestra de que "no hay ignorantes absolutos ni sabios absolutos: hay hombres que, en comunicación, buscan saber más" (Freire, 2009: 45).

Asumir la concepción pedagógica de Freire en la educación superior requiere de una constante reflexión entre la cultura academicista tradicional y la misión educativa para el desarrollo sostenible, que permita su conversión en una práctica de liberación: "La educación como práctica de la libertad, al contrario de aquella que es práctica de la dominación, implica la negación del hombre abstracto, aislado, suelto, desligado del mundo, así como la negación del mundo como una realidad ausente de los hombres" (Freire, 2009: 38).

La experiencia práctica de servicio comunitario de Gregorutti y Charles-Marcel (2013), unida a la de Sánchez y Caldera (2013), concibe a la comunidad como un espacio de aprendizaje basado en relaciones interinstitucionales, intereses políticos y gubernamentales, con un objetivo común.

Sánchez y Caldera (2013: 77) también muestran coincidencia con García (2013), al proponer de manera similar que: "Los ambientes de aprendizaje, entonces, deben garantizar que los propios actores confronten sus experiencias con la realidad, con las 
interpretaciones o puntos de vista de los otros, lo que favorece el diálogo, el debate y la investigación emanada de los espacios públicos".

En Pérez-Pérez (2014) y Restrepo-Salazar et al. (2015), la acción cultural es transversal a sus propuestas. En el primer caso se menciona una metodología participativa que es viable a través de la animación sociocultural; mientras que en el segundo, la extensión universitaria es la estrategia que se asume. Ambos autores centran su interés en la implicación colectiva de los participantes de la relación universidad-comunidad.

Ávila et al. (2016: 764) abordan la experiencia de educación superior intercultural en México, explicitando que: "En el centro de la institución se encuentra la investigación sobre las culturas y las lenguas de los pueblos a los que sirve. Desde ahí se permea toda la vida docente y de servicio de la universidad".

Esta propuesta de la educación superior intercultural se acerca más a la concreción en la práctica social de la dimensión cultural del desarrollo. Es expresión de los principios rectores de la Convención sobre la Protección y la Promoción de la Diversidad de las Expresiones Culturales (UNESCO, 2005: 4), que en síntesis reconoce que: "La diversidad cultural es una gran riqueza para las personas y las sociedades. La protección, la promoción y el mantenimiento de la diversidad cultural son una condición esencial para un desarrollo sostenible en beneficio de las generaciones actuales y futuras".

El trabajo de Ávila et al. (2016: 768-769), aborda un modelo educativo donde:

la vinculación comunitaria es el eje articulador que permite conjuntar las bases teóricas, aprendidas y ejercitadas en el aula, de todas las asignaturas con una parte práctica que posibilita un diálogo intercultural y el desarrollo de alternativas junto con las comunidades. Ello se hace a través de talleres participativos donde se busca la presencia de representantes de la vida social de las diversas comunidades rurales de la región.

El modelo educativo que estos autores presentan tiene una estructura por semestres que se organiza desde los aportes de la IAP (cuadro 3), donde los semestres del primero al tercero son predominantemente reflexivos; los semestres del cuarto al séptimo son mayoritariamente de acción, y el octavo representa el cierre de un ciclo de reflexión-acción-reflexión.

Aun cuando el modelo educativo de vinculación comunitaria es una de las experiencias de mayor interés para la investigación, se reconocen algunas limitaciones de este: a) visión positivista en el estudio de las comunidades; b) visión piadosa o asistencialista del trabajo comunitario; c) visión proyectista que no atiende de manera integral la problemática comunitaria; d) la perspectiva basada en prácticas de educación popular centrada en campesinos e indígenas, que generó temores y prejuicios en su implementación (Ávila et al., 2016: 771).

Para Castro et al. (2016), la vinculación entre trabajo comunitario y formación académica se concibió como una estrategia que permitiera articular los contenidos y lecturas de los planes de estudio, el desarrollo de habilidades en investigación, la incorporación de los saberes comunitarios y el sentido de responsabilidad social con las comunidades.

Castro et al. (2016: 749), entienden la vinculación comunitaria como: "Una estrategia formativa que permite crear relaciones de enseñanza-aprendizaje con sentido para todos los actores del proceso (estudiantes, profesores, comunidades)".

La investigación de Castro et al. (2016) reconoce el valor del vínculo entre saber académico y saber popular, unidos a su vez por la actividad investigativa que desarrollan los estudiantes. Para estos autores, las universidades tienen la responsabilidad de promover, desarrollar y generar cultura, tanto en su interior como en el exterior. 


\section{Cuadro 3. Modelo educativo de vinculación universidad-comunidad}

\begin{tabular}{|c|c|c|}
\hline Semestres & Propósitos & Descripción de las etapas \\
\hline Primero & Sensibilización. & Los estudiantes se acercan e insertan en los ámbitos rurales. \\
\hline Segundo & $\begin{array}{l}\text { Asimilación reproductiva y } \\
\text { productiva del conocimiento }(\mathrm{I}) \text {. }\end{array}$ & $\begin{array}{l}\text { Los estudiantes se apropian de un conjunto de herramientas conceptuales y metodológicas que les } \\
\text { permiten trabajar de manera participativa en y desde las comunidades. }\end{array}$ \\
\hline Tercero & $\begin{array}{l}\text { Asimilación reproductiva y } \\
\text { productiva del conocimiento (II). }\end{array}$ & $\begin{array}{l}\text { Los estudiantes integran los conocimientos de los pueblos originarios con aquellos saberes que } \\
\text { provienen del mundo académico. }\end{array}$ \\
\hline Cuarto & Diagnóstico comunitario integral. & $\begin{array}{l}\text { La comunidad universitaria debe trabajar, de manera participativa, en un autodiagnóstico que le } \\
\text { permita ubicar sus problemáticas sociales, culturales, ambientales y económicas. }\end{array}$ \\
\hline Quinto & $\begin{array}{l}\text { Planificación de propuestas de } \\
\text { acción comunitaria. }\end{array}$ & $\begin{array}{l}\text { Los estudiantes trabajan con herramientas conceptuales y metodológicas para construir con las } \\
\text { comunidades planes participativos a través de los cuales se pueda colaborar con las sociedades } \\
\text { rurales en propuestas de acción e incidencia hacia el buen vivir. }\end{array}$ \\
\hline Sexto & $\begin{array}{l}\text { Diseño de propuestas de acción } \\
\text { comunitaria. }\end{array}$ & $\begin{array}{l}\text { Los estudiantes avanzan en el trabajo con diversas propuestas comunitarias, para ello se apropian } \\
\text { de elementos de la matriz del marco lógico y del diseño de proyectos. }\end{array}$ \\
\hline Séptimo & $\begin{array}{l}\text { Ejecución de propuestas de } \\
\text { transformación comunitaria. }\end{array}$ & $\begin{array}{l}\text { Los estudiantes construyen procesos de transformación y empoderamiento de las comunidades en } \\
\text { alianza con la Universidad. }\end{array}$ \\
\hline Octavo & Evaluación. & $\begin{array}{l}\text { Los estudiantes realizan una evaluación participativa de la experiencia de vinculación comunitaria } \\
\text { que permita a los estudiantes y a la comunidad sistematizarla y aprender de ella para una mejora de } \\
\text { sus prácticas. }\end{array}$ \\
\hline
\end{tabular}

Fuente: elaboración propia.

Las experiencias de vinculación universidad-sociedad-comunidad de los autores presentados, defienden la unidad entre protagonistas del proceso educativo (educandos, educadores y comunidad), con base en el diálogo y la investigación-acción participativa (IAP), hecho que reafirma la importancia de la unidad dialéctica entre acción, reflexión y su devolución hacia una práctica transformadora, como uno de los principios necesarios de la misión educativa de la educación superior.

Hernández (2009) señala que la IA como método de indagación social es una metodología democrática, basada en el respeto a la individualidad, la apertura a la diversidad, la riqueza de técnicas y procedimientos posibles, así como la flexibilidad de enfoques. Parte de los postulados que la hacen diferente de la investigación tradicional son: 1) la comunidad identifica el problema o necesidad a resolver; 2) el objeto de la investigación está en el mejoramiento de las condiciones humanas mediante el cambio de una situación desfavorable; 3) la naturaleza de la situación problemática se encuentra en la esfera del mundo humano de ideas y creencias, en la conducta humana reflexiva; 4) el investigador debe formar parte de la experiencia colectiva, su relación con el contexto se da a través de las percepciones.

Los saberes aportados por la educación popular (EP) y por la IA promueven el valor de lo comunitario, 
de lo grupal y lo individual. Las expresiones de la cultura se convierten en el eje dinamizador del análisis de problemáticas sociales, teniendo en cuenta la necesidad de:

- Conocer aspectos teóricos y metodológicos para identificar y caracterizar problemáticas socioculturales así como la formulación de explicaciones.

- Elaborar diagnósticos integrales acordes con la situación real de la población y su entorno.

- Diseñar, planificar y trazar líneas de acción integrales.

- Participar en la toma de decisiones de los actores sociales.

- Implementar acciones de educación y capacitación social.

- Rescatar la cotidianidad y la historia local o comunitaria.

- Impulsar el desarrollo de los sectores populares en las estrategias de desarrollo.

- Lograr que los servicios sociales sean alcanzados por los sectores populares a través de procesos de gestoría social.

En sentido general, los trabajos consultados son una muestra de la concienciación individual y colectiva de la misión educativa de la educación superior para el desarrollo sostenible en las universidades; sin embargo, han demostrado que la actividad transformadora no puede ir atada a viejas prácticas de intervención comunitaria, sino que tiene que renovarse constantemente en función de la sostenibilidad de la solución de problemáticas transdisciplinares, incluso en aquellas experiencias de acción comunitaria con aportes cuyo valor ha sobrevivido en el tiempo.

\section{Una propuesta emergente para la misión educativa de la educación superior}

El estudio de las experiencias del vínculo universidad-sociedad-comunidad permitió identificar que la dimensión cultural del desarrollo es un concepto que requiere ser incorporado a los análisis teórico-metodológicos, considerando que "la cultura ha sido objeto de estudio como elemento necesario para el pleno desarrollo de las personas y las comunidades" (UNESCO, 2010: 4).

Aun cuando se consideran valiosos los aportes teórico-metodológicos de las investigaciones realizadas, es criterio de la autora que, tratándose de la misión educativa de la educación superior, se requiere el análisis del concepto de cultura y su significación para el desarrollo sostenible.

La comprensión de la cultura como identidad de las sociedades, la convierte en eje dinamizador del desarrollo. En el Informe Final de la Conferencia Mundial sobre las Políticas Culturales se reconoce que "toda política de desarrollo debe enfocar al hombre en su totalidad, es decir, en toda su dimensión personal, sin olvidar sus aspectos étnicos, religiosos y espirituales y sus dimensiones sociales" (UNESCO, 1982: 11).

La cultura en su sentido más amplio está compuesta por "el conjunto de los rasgos distintivos, espirituales y materiales, intelectuales y afectivos que caracterizan una sociedad o un grupo social" (UNESCO, 1982: 43). Así también, "El desarrollo debe fundarse en los valores culturales de las sociedades, manifestando el mayor respeto por la personalidad de cada individuo. Debe estar centrado en la persona humana y en las comunidades que ésta integra" (ibid:: 11).

El vínculo universidad-sociedad-comunidad, no debe concebirse ajeno al concepto de dimensión cultural del desarrollo, pues ésta:

no es una dimensión como cualquier otra, no es uno de tantos factores que tomados en su conjunto constituyen los elementos del desarrollo. Por el contrario, es el factor fundamental del desarrollo, la referencia básica por la que se miden todos los demás factores. Por lo tanto, esto significa que no puede existir un desarrollo realmente satisfactorio y sostenible que 
no reconozca y utilice la fuerza vitalizadora de la cultura y haga caso omiso de los estilos de vida, sistemas de valores, tradiciones, creencias, conocimientos y aptitudes de la comunidad (UNESCO, 1994: 7).

En una propuesta de vinculación universidadcomunidad, centrada en la dimensión cultural del desarrollo, se valora el enfoque endógeno del desarrollo, como una condición sine qua non del trabajo comunitario, pues a través de él se asigna a la población beneficiaria una función activa en su propio desarrollo. Igualmente, se exige tener en cuenta el contexto sociocultural, las condiciones específicas vinculadas a una determinada cultura, la capacidad creadora de la comunidad, sus valores, potencialidades y sus formas de expresión cultural (UNESCO, 1994).

Los aspectos de la identidad cultural que la UNESCO reconoce, deben ser entendidos desde su carácter dialéctico pues: "La cultura no es un conjunto estático de valores y prácticas. Se recrea constantemente en la medida en que las personas cuestionan, adaptan y redefinen sus valores y prácticas ante el cambio de la realidad y el intercambio de ideas" (PNUD, 2004: 4).

Ese carácter dialéctico de la cultura conlleva a que sea "uno de los principales motores del desarrollo" (UNESCO, 2005: 4), y en su vínculo con la educación "es un elemento importante en la conciliación de las identidades grupales en un marco de cohesión social" (UNESCO, 2006: 12). En este sentido, en la investigación se propone pensar el servicio comunitario de la universidad desde un enfoque sociocultural.

El término sociocultural en su función adjetiva explicita esta unidad entre sociedad y cultura, que se traduce en el valor de la segunda para el desarrollo social, en la necesidad de interpretar y transformar las problemáticas sociales desde la comprensión de la complejidad de las prácticas humanas, la participación y el respeto a la identidad cultural.

Sobre el mismo término, Martín (2005: 45) plantea que éste "destaca el carácter social de la cultura o el carácter cultural de los fenómenos sociales". Por su parte, para Casanova y Carcassés (2007: 54): "El prefijo "socio" resulta un elemento compositivo que recalca la proyección social de las acciones que se orientan a desarrollar la cultura, en la medida en que inciden en el comportamiento de la sociedad en su conjunto, con el propósito de favorecer la creatividad, la integración y la participación de individuos, grupos y comunidades, en programas sociales".

A diferencia de Casanovas y Carcassés, los enfoques internacionales con los que coincide la autora de este artículo, consideran a la cultura como impulsora del desarrollo social. El uso del prefijo "socio" no debe interpretarse únicamente como reflejo de una participación social en función del desarrollo cultural.

Freyre (2013: 5) plantea: "El término sociocultural invita a pensar en dos realidades objeto de estudio científico: la sociedad y la cultura, lo social y lo cultural"; en calidad de realidad o enfoque, apunta al entronque entre la sociología y la antropología cultural.

Aun cuando en el plano teórico la sociología y la antropología puedan considerarse fuentes epistemológicas del término "sociocultural", igualmente se considera que el discurso actual sobre la dimensión cultural del desarrollo obliga a repensar "lo sociocultural" como una categoría transdisciplinar con diversas fuentes teóricas (filosófica, psicológica, ecológica, enfoque de sistema, entre otros).

Del análisis de los documentos de la UNESCO, en la investigación se considera que lo social se compone de las esferas económica, política, cultural, científica, tecnológica, educativa y ambiental; de ahí que al hacer uso del término "sociocultural" se pretende sintetizar la complejidad de interrelaciones que integran a la sociedad, enfatizando el valor de la identidad cultural y de la participación individual y colectiva de las personas, para su desarrollo presente y futuro.

El término sociocultural desde su función adjetiva debe ser usado como una categoría transdisciplinar 
que hace alusión a la comprensión de los nexos complejos que ocurren a diferentes niveles de una sociedad (institucional, comunitario e individual), que reflejan la conexión entre lo social y lo cultural como un todo, que permiten comprender el desarrollo y su transformación, en un sentido general (Martínez, 2017). A partir de este criterio, aquí se asume la misión educativa de la educación superior desde una concepción de trabajo sociocultural comunitario.

El término trabajo sociocultural comunitario (TSC) pudiera parecer un juego de palabras que parte de los conceptos de trabajo social, trabajo comunitario y acción cultural; el empleo del prefijo "socio" pudiera considerarse tautológico, puesto que las fronteras entre la sociedad y la cultura son más una cuestión epistemológica que práctica si tomamos en consideración que "la cultura nutre la trama social y la importancia de su papel es tan determinante que la cultura puede finalmente confundirse con la propia vida" (UNESCO, 1982: 8). Al emplear el término trabajo sociocultural comunitario, no se niegan las experiencias teóricas y metodológicas del trabajo social y comunitario, así como de la acción cultural, sino que se pretende un diálogo entre los aportes más valiosos para un desarrollo sostenible.

El término TSC intenta brindar la mirada holística que muchas veces ha estado ausente en las prácticas de intervención social y comunitaria. Aboga por el desarrollo sostenible tomando como principio que: "La libertad cultural constituye una parte fundamental del desarrollo humano [...] Es necesario que la gente cuente con la libertad de participar en la sociedad sin tener que desprenderse de los vínculos culturales que ha escogido" (PNUD, 2004: 1).

Con el TSG se pretende que la acción cultural, en un sentido integral, sirva de eje central para la participación social de diversos actores (comunidad, instituciones sociales y culturales, empresas, organizaciones, etcétera), en el desarrollo endógeno de una comunidad.

\section{Principales ideas del trabajo sociocultural comunitario en la Universidad}

El TSC representa una propuesta para la misión educativa de la educación superior en relación con el desarrollo sostenible. Es una actividad que no restringe perfiles profesionales, pues:

La apropiación de la información y su transformación en conocimiento por parte de los ciudadanos es un acto cultural. Por lo tanto, el acceso sin distinciones a los medios de expresión, tecnológicos y de comunicación y la constitución de redes horizontales fortalece y alimenta la dinámica de las culturas locales y enriquece el acervo colectivo de una sociedad que se basa en el conocimiento (Agenda 21, 2004: 9).

La educación superior tiene la responsabilidad social de promover espacios que permitan la democratización de la ciencia y la tecnología, así como la alfabetización científico-tecnológica a partir de una labor extensionista con las comunidades; de ahí que debe responder al compromiso de: "Fomentar los programas dirigidos a divulgar la cultura científica y la tecnología entre todos los ciudadanos; especialmente, si se considera que las posibles aplicaciones de los nuevos conocimientos científicos generan cuestiones éticas, sociales, económicas y políticas que son de interés público" (Agenda 21, 2004: 11).

El TSG se define como la estrategia de formación profesional en la universidad y consiste en la planificación, organización, ejecución, control, evaluación y sistematización de un sistema de acciones que durante los periodos de duración de los procesos formativos de pregrado y posgrado, permita a estudiantes, profesores y personal no docente, participar en la solución sostenible de problemáticas comunitarias.

Para que el TSC sea viable, los docentes y personal administrativo universitario deben tomar en consideración un grupo de acciones educativas sustentadas en los principios de: 1) vinculación de la educación con la vida, el medio social y el trabajo; 
2) formación para la investigación sociocultural; 3) participación social como eje transversal de la actividad transformadora; 4) unidad entre la actividad educativa y la comunicación (cuadro 4).

La vinculación de la educación con la vida, el medio social y el trabajo, representa un principio para lograr la participación de los estudiantes en el TSG. Al asumirlo se coincide con Morin (1999) cuando expresa la necesidad de que la educación promueva la inteligencia general que implica la contextualización del conocimiento, el reconocimiento de la complejidad entre el todo y las partes, que permita la integración de saberes históricamente fragmentados. De igual forma, se reconoce el valor de la cultura para el desarrollo social y el bienestar humano, desde una visión holística que tiene en cuenta su multidimensionalidad económica, filosófica, política, sociológica, antropológica, ambiental, etcétera, así como la complejidad de sus interrelaciones.

\section{Cuadro 4. Principios y acciones educativas del trabajo sociocultural comunitario en la Universidad}

\begin{tabular}{|c|c|}
\hline Principios & Acciones educativas \\
\hline $\begin{array}{l}\text { Vinculación de la educación con la } \\
\text { vida, el medio social y el trabajo. }\end{array}$ & $\begin{array}{l}\text { - } \quad \text { Diagnosticar el conocimiento precedente de los estudiantes sobre el valor social de su profesión. } \\
\text { - } \text { Problematizar sobre el impacto de la profesión en la sociedad en unidad con la dimensión curricular y } \\
\text { extracurricular. } \\
\text { - } \quad \text { Contextualizar la formación de los estudiantes, logrando una unidad efectiva entre la teoría y la práctica. }\end{array}$ \\
\hline $\begin{array}{l}\text { Formación para la investigación } \\
\text { sociocultural. }\end{array}$ & $\begin{array}{l}\text { - Diagnosticar las competencias investigativas de los estudiantes. } \\
\text { - Diseñar competencias investigativas acordes con la Zona de Desarrollo Próximo (ZDP) de los estudiantes. } \\
\text { - Propiciar una educación científica. } \\
\text { - Potenciar el trabajo interdisciplinar y transdisciplinar entre profesores y estudiantes. }\end{array}$ \\
\hline $\begin{array}{l}\text { Participación social como } \\
\text { eje transversal de la actividad } \\
\text { transformadora. }\end{array}$ & $\begin{array}{l}\text { - Diagnosticar las habilidades sociales de los estudiantes. } \\
\text { - Diseño de actividades curriculares y extracurriculares a partir de los resultados del diagnóstico. } \\
\text { - Incorporación de la comunidad como protagonista del proceso educativo de los estudiantes. } \\
\text { - Evaluación conjunta del proceso de formación profesional de los estudiantes. }\end{array}$ \\
\hline $\begin{array}{l}\text { Unidad entre la actividad educativa y } \\
\text { la comunicación. }\end{array}$ & $\begin{array}{l}\text { - Diagnosticar las competencias comunicativas de los estudiantes. } \\
\text { en el diagnóstico. } \\
\text { - Incorporar en la relación educando-educador la cultura del diálogo y la participación en su proceso de } \\
\text { enseñanza aprendizaje. }\end{array}$ \\
\hline
\end{tabular}


Lo anterior implica un vínculo entre las acciones que se realizan tanto en la dimensión curricular como extracurricular, de manera tal que se propicie la repetición de acciones, su reforzamiento y perfeccionamiento, en correspondencia con el objetivo que se proponga y las necesidades de los estudiantes.

Los profesores tienen que vincular su mensaje educativo con la vida, con el medio social y el trabajo. Para ello es importante la participación de los estudiantes en los contextos socioculturales, el intercambio con las agencias y agentes socializadores que pueden estar implicados en un proceso de investigación sociocultural comunitario. Con este fin, es necesario el uso de métodos de la profesión en un proceso de enseñanza problémico, donde el estudiante sea un protagonista activo y transformador de su aprendizaje.

La formación para la investigación sociocultural es otro de los principios educativos a considerar. Debe estar en correspondencia con la lógica de la ciencia para la solución de problemas socioculturales. No se trata de una formación en contradicciones con la investigación científica, es un proceso que implica la búsqueda de soluciones desde un enfoque transdisciplinar.

En consecuencia con los conceptos de la UNESCO sobre cultura, política cultural y dimensión cultural del desarrollo, los problemas socioculturales expresan dificultades o contradicciones cuya solución requiere de un proceso de investigación, teniendo en cuenta la capacidad creadora de cada persona y comunidad, así como su participación en el desarrollo social.

Los problemas socioculturales están presentes en espacios grupales, comunitarios, institucionales u organizacionales; pueden expresarse a nivel microsocial y macrosocial, donde se atienden los siguientes aspectos: estimular las actitudes creadoras y la vida cultural; fomentar la salvaguardia y revalorización del patrimonio; preservar los valores espirituales, sociales y humanos de cada sociedad; estimular las actividades culturales como expresión de las identidades; mejorar el acceso de todos a la vida cultural y su participación en ésta; favorecer la plena expansión de la creación y de la creatividad, y fortalecer los intercambios y cooperación cultural.

Ante la realidad sociocultural, la investigación cultural y la social se configuran en una investigación que permite realizar análisis cualitativos y cuantitativos de la vida de los sujetos y sus expresiones culturales. La experiencia de una investigación social permite, según Galeana de la O (2006), “obtener nuevos conocimientos en el campo de la realidad social o bien estudiar una situación para diagnosticar necesidades y problemas a efecto de aplicar los conocimientos con fines prácticos". Y la investigación cultural se convierte en un proceso de gestión que desde el punto de vista de Eduardo y Linares (2009) aporta "conocimientos científico y otros saberes que desempeñan los roles de las políticas sociales y factor de transformación social".

La cultura se investiga para analizar, describir, explicar, predecir o transformar una realidad social, así como para hallar acciones dinamizadoras, reflexivas y creativas en la que los sujetos participen activamente, comprometidos e identificados en la construcción del saber necesario para la aplicación de políticas sociales y de desarrollo. De esta manera, la investigación sociocultural debe comprenderse desde prácticas en las que realmente los sujetos sean protagonistas del estudio y transformación de su realidad a partir de experiencias participativas y dialógicas.

A consideración de Eduardo y Linares (2009), el proceso de investigación cultural en el que existe una participación consciente, activa y responsable de los sujetos implicados, en la construcción de un saber que complementa los modos tradicionales y academicistas de indagar en la realidad con saberes populares o alternativos, para la solución de problemáticas, adquiere una dimensión sociocultural.

Formar para la investigación sociocultural, en consecuencia con el método científico, conlleva el tránsito de los estudiantes por las siguientes etapas: 
tomar conocimiento de problemas que otros pueden haber pasado por alto (problematizar); insertarlos en un cuerpo de conocimiento, e intentar resolverlos con el máximo rigor (teorizar y comprobar) de manera tal que permita enriquecer su conocimiento (Bunge, 1966).

Con base en el método científico, el estudiante en su formación como investigador sociocultural deviene "un problematizador por excelencia" (Bunge, 1966: 191), en la medida que identifica dificultades o contradicciones en las prácticas y políticas culturales, que representan los problemas de su profesión.

La participación social como eje transversal de la actividad transformadora es un principio que se fundamenta en el reconocimiento del papel transformador y creativo de los actores sociales (estudiantes, profesores, comunidad e instituciones que la integran), teniendo en cuenta las dimensiones de la participación: política, cultural y pedagógica. Responde a principios universales de igualdad, equidad, paz, democracia, libertad cultural, entre otros. Es la práctica opuesta a modelos autoritarios o paternalistas, en los que imperan actitudes pasivas y dependientes ante los problemas de las personas.

El trabajo sociocultural comunitario debe ser concienciado por sus beneficiarios. Los estudiantes y los profesores no deben reproducir prácticas desfavorables a la sostenibilidad, porque cuando cese su participación en la vida de la comunidad la transformación alcanzada puede desaparecer.

La participación social es un proceso que se educa y, según Alejandro (2008: 77): "La participación precisa, sobre todo, un cambio cualitativo en cada uno de nosotros. Es, ni más ni menos, el proceso por el cual se quiebra la relación de sumisión entre los actores involucrados en un proceso: educando-educador, investigadores-investigados".

La unidad entre la actividad educativa y la comunicación es un principio que resalta el valor de la interacción de los estudiantes con el TSC, donde tienen una incidencia significativa las relaciones que se establecen entre educandos, profesores, tutores de una práctica laboral y agentes socializadores que intervienen en el estudio y solución de problemas socioculturales.

Para el desarrollo de este principio, los docentes deben planificar un proceso participativo, dialógico, reflexivo, problematizador, dialéctico y contextualizado, ya que ellos cumplen la función de guías, orientadores y facilitadores de las relaciones estudiante-comunidad, en el que están presentes un sistema de conocimientos, habilidades y valores. Estos llegan a los estudiantes a través de la comunicación oral, corporal y el ejemplo personal del profesor, como parte de las estrategias que influyen en la transformación del educando.

En este principio los métodos juegan un papel importante puesto que permiten la organización de la actividad y la comunicación que se desarrollan en el proceso, permitiendo que el profesor llame la atención constantemente sobre la necesidad de solucionar problemas socioculturales y las vías para su realización.

El principio permite reconocer el alto valor formativo de la actividad y la comunicación en el contexto grupal, ya que propician la implicación personal y vivencial en el proceso de formación para la investigación sociocultural. En este sentido, la apropiación del contenido no sólo ocurre en el interior de los estudiantes, sino que tiene su expresión externa, social y comunicativa.

\section{Etapas para el desarrollo del trabajo sociocultural comunitario (TSC)}

Las acciones educativas que se presentaron, tienen la intención de que el TSG se planifique teniendo en cuenta las potencialidades de los estudiantes y del proceso de enseñanza-aprendizaje. En este sentido, las etapas que se proponen tienen el propósito de articular estas acciones educativas, que resultan generales, de acuerdo con los diferentes momentos en que se concibe el trabajo con las comunidades desde un enfoque sociocultural. 
El trabajo sociocultural comunitario se concibe en cinco etapas (véase cuadro 5); cada etapa debe tener un desarrollo en lo curricular y extracurricular. De igual manera requiere de un diagnóstico de la ZDP de los estudiantes para enfrentarse a un nuevo objetivo educativo.

En la primera etapa, identificación de problemas socioculturales, los estudiantes deben participar en el contexto de investigación teniendo en cuenta las siguientes acciones del trabajo comunitario: observar el contexto, caracterizar el contexto, determinar contradicciones o dificultades socioculturales en correspondencia con la política cultural del país y sus comunidades, jerarquizar las contradicciones o dificultades halladas, plantear problemas socioculturales y comunicar los resultados del proceso.
Esta etapa se caracteriza por: a) la preparación del trabajo sociocultural comunitario, a partir de la presentación de los servicios que la universidad puede ofrecer a la comunidad, y la recepción de demandas por parte de las autoridades académicas, así como la conciliación con líderes comunitarios y gubernamentales de la participación de estudiantes y profesores, en la atención a sus problemáticas; b) la determinación de tutores de la universidad y la comunidad; c) la determinación de la cultura universitaria que se necesita transferir a la comunidad, expresada en los saberes académicos que promueve y genera la universidad; d) la introducción de métodos cualitativos que favorezcan la familiarización de estudiantes y profesores con la comunidad: etnografía y biografía o historia de vida; e) la descripción de las

\section{Cuadro 5. Etapas del trabajo sociocultural comunitario}

\begin{tabular}{|l|l|}
\hline \multicolumn{1}{|c|}{ Etapa } & \multicolumn{1}{c|}{ Objetivos educativos } \\
\hline Identificación de problemas socioculturales. & $\begin{array}{l}\text { Desarrollar competencias para la identificación de contradicciones entre la teoría que se estudia } \\
\text { y la vinculación de los estudiantes en las comunidades. }\end{array}$ \\
\hline Diseño del proceso de investigación sociocultural. & $\begin{array}{l}\text { Desarrollar competencias para el diseño del proceso de investigación sociocultural de manera } \\
\text { participativa. }\end{array}$ \\
\hline Ejecución de la solución al problema sociocultural. & $\begin{array}{l}\text { Desarrollar competencias para acompañar la ejecución de la solución a problemas } \\
\text { socioculturales en comunidades. }\end{array}$ \\
\hline Evaluación de la solución al problema sociocultural. & $\begin{array}{l}\text { Desarrollar competencias para la evaluación parcial y final de los resultados alcanzados en la } \\
\text { comunidad durante la solución de su problema sociocultural. }\end{array}$ \\
\hline
\end{tabular}

Fuente: elaboración propia. 
contradicciones que se observan en la comunidad a partir de la teoría estudiada.

La segunda etapa, el diseño del proceso de investigación sociocultural, permite a los estudiantes y profesores participar junto a la comunidad en la organización y planificación del proceso de investigación, a partir del diseño de componentes teóricos y metodológicos, que le ofrecen rigurosidad a los resultados que se obtienen y propician una unidad entre la teoría y la práctica para la solución del problema sociocultural.

En esta etapa deben considerarse las siguientes acciones: plantear un problema de investigación sociocultural, determinar los componentes teóricos y metodológicos del diseño, diagnosticar el problema sociocultural, recopilar datos, analizar la información, proponer una solución al problema sociocultural y comunicar los resultados.

Esta etapa se caracteriza por: a) la participación de los estudiantes y profesores en la elaboración de la monografía comunitaria; b) la introducción del enfoque mixto de la investigación (unidad entre lo cualitativo y cuantitativo), con predominio de los métodos de IAP y la Fenomenología; c) la determinación del saber comunitario ancestral y actual, que permite la solución del problema investigado.

Teniendo en cuenta que, con base en la ciencia, es la práctica humana la que "demuestra la correspondencia de nuestras representaciones a la naturaleza objetiva de las cosas que percibimos" (Lenin, 1976: 129), se considera que en el proceso educativo para el desarrollo sostenible, los estudiantes deben poseer competencias para la comprobación de la propuesta de solución a los problemas socioculturales, a partir de su ejecución y evaluación.

La tercera etapa, ejecución de la solución al problema sociocultural, es una etapa que implica la aplicación de las acciones transformadoras que se proponen por la comunidad. Para su realización es necesario desarrollar lo siguiente: observar las acciones que se ejecutan, caracterizar el proceso de ejecución, determinar métodos y técnicas que permitan la valoración de la ejecución, aplicar métodos y técnicas de investigación sociocultural, recopilar los datos, interpretar la información, valorar la ejecución y comunicar los resultados.

La evaluación de la solución al problema sociocultural, es la cuarta etapa, permite comprobar en la práctica el cumplimento de los objetivos de las acciones del trabajo sociocultural comunitario. Para su realización es necesario participar en el contexto donde se aplica la solución al problema de investigación sociocultural, fundamentar teórica y metodológicamente el proceso de evaluación, aplicar métodos, técnicas y procedimientos de investigación con un enfoque participativo, valorar la información obtenida y comunicar los resultados.

La ejecución y la evaluación son etapas que se caracterizan por: a) la sistematización de competencias de etapas anteriores; b) la reflexión sistemática de la unidad teoría-práctica, c) el predominio de métodos y técnicas participativas con enfoque transdisciplinar, d) la evaluación del vínculo universidad-sociedadcomunidad y la proyección de acciones para nuevas etapas de trabajo conjunto.

La última etapa, sistematización de la experiencia investigativa, integra a las anteriores y en ella se propone la construcción grupal e individual de los resultados alcanzados en el trabajo sociocultural comunitario. Se caracterizar por: a) la reflexión profunda de la unidad teoría-práctica sociocultural comunitaria desarrollada; b) la generación de nuevos aportes teóricos y metodológicos; c) la publicación y presentación de los resultados en contextos académicos y no académicos; d) la promoción de acciones de colaboración nacional o internacional.

Como se puede apreciar, la comunicación de los resultados se integra en todas las etapas. Su intención no debe orientarse solamente a la socialización de resultados en un contexto académico, sino en espacios extracurriculares y de extensión universitaria, donde estén presentes la comunidad, las agencias y agentes socializadores implicados en el TSC desarrollado. 


\section{Conclusiones}

Las experiencias de trabajo comunitario universitario predominan sobre las restantes del vínculo universidad-sociedad-comunidad, apreciándose en ellas la existencia de acciones que, aunque valiosas para la misión educativa de la educación superior, aún reproducen prácticas negativas con un carácter asistencialista e interventor-colonialista, ya que la cultura académica positivista, predomina sobre las necesidades sentidas de las comunidades y la participación en la solución de sus problemas desde la valoración de sus saberes popular-ancestral y sus potencialidades físico-geográficas y socioculturales.

El trabajo comunitario universitario se concibe mayoritariamente como un servicio de la responsabilidad social de la educación superior; sin embargo, su concepción se centra más en los intereses académicos que en la transformación de una problemática comunitaria.

La concepción del trabajo comunitario universitario es insuficiente en la articulación de actores y agencias sociales, ya sean públicas o privadas, gubernamentales y políticas. En este sentido se considera que la educación superior tiene el reto de fortalecer la implicación de los gobiernos locales en la atención al vínculo universidad-sociedad-comunidad.

La IAP es la metodología que con mayor frecuencia fundamenta las experiencias de trabajo comunitario universitario. Sobre esta realidad se considera que para el trabajo con la comunidad se requiere incorporar el enfoque mixto de investigación y los métodos cualitativos de etnografía, fenomenología y biografía o historia de vida.

El reconocimiento de la cultura como eje dinamizador de la participación y transformación social es un aspecto débil en las experiencias de trabajo comunitario universitario, de ahí que se propone incorporar el enfoque sociocultural para su realización, hecho que implica la valoración de los conceptos de cultura y dimensión cultural del desarrollo.

El trabajo sociocultural comunitario es la propuesta de una estrategia educativa para la educación superior que permita formar al estudiante universitario (de perfiles diferentes), en la sensibilización y atención por los problemas comunitarios de su nación. En él se pretende que los estudiantes desarrollen competencias de acción local a problemáticas globales.

El trabajo sociocultural comunitario es una estrategia educativa que puede llegar a limitarse si no se concibe desde la acción transdisciplinar universitaria. Lo ideal en este sentido sería incorporar diversos estudiantes y profesores en la solución de una problemática comunitaria, lo cual permite educarlos en el análisis de la realidad desde la complejidad de sus interrelaciones.

La concepción del trabajo sociocultural comunitario requiere que su organización y planificación se sustenten en la correlación de las acciones y objetivos educativos, con sus etapas de identificación, diseño, ejecución, evaluación y sistematización. 


\section{Referencias}

Agenda 21 de la Cultura (2004), <http://www. agenda 21 culture.net/index.php/docman / agenda21/222-ag21es/file> [Consulta: enero de 2017].

Alarcón, R. (2016), Universidad innovadora por un desarrollo sostenible: mirando al 2030, Cuba, Editorial Félix Varela.

Alejandro, M. (2008), "La participación: reconceptualizando el tema", en Armando Chaguaceda (comp.), Participación y espacio asociativo, Cuba, Publicaciones Acuario, pp. 69-77.

Ávila, L. E., A. Betancourt, G. Arias y A. Ávila (2016), "Vinculación comunitaria y diálogo de saberes en la educación superior intercultural en México", en Revista Mexicana de Investigación Educativa, vol. 21, núm. 70, <http://web.a.ebscohost.com/ehost/pdfviewer/ pdfviewer ?vid $=5 \&$ sid $=645$ e 3 a $75-$ ec $69-44$ a $9-837 \mathrm{~d}-$ 3cee0ae9fcf8\%40sessionmgr4010\&hid=4114> [Consulta: enero de 2017].

Bunge, M.A. (1966), La investigación científica. Su estrategia y su filosofia, Cuba, Editorial de Ciencias Sociales.

Casanovas, A. y A. I. Carcassés (2007), "Acciones dinamizadoras de la participación de los cubanos en la cultura", en S. Almazán del Olmo y P. Torres, Panorama de la cultura cubana, Antología, Cuba, Editorial Félix Varela, pp. 53-79.

Castro M. L., M. J. Rodriguez y E. Urteaga (2016), "Abrir las aulas: el vínculo entre docencia, investigación y vinculación comunitaria”, en Revista Brasileira de Educação, vol. 21, núm. 66, julio-septiembre, pp. 737-758.

Eduardo, E. e I. Linares (2009), "Investigación cultural y política social en la Cuba del siglo XXI. Una relación retadora", Ponencia presentada en el II Simposio internacional Las Ciencias Sociales y los actores de cambio social: alternativas de desarrollo social, Cuba, Centro de Investigaciones Psicológicas y Sociológicas (CiPS, en CD-R. ISBN 978959237 267-2, pp. 1-26.

Freire, P. (2009), Pedagogía del oprimido, Cuba, Editorial Caminos.

Freyre, E. F. (2013), “¿Qué son los estudios socioculturales?”, en B. Rojas y L. Amaury, (comps.), Lo sociocultural, un trabajo pendiente, La Habana, Editorial de Ciencias Sociales, pp. 3-52.

Galeana de la O, S. (2006), Promoción social: una opción metodológica, México, Plaza y Valdés.

García, T. (2013), "Experiencias y posibilidades de articulación entre universidad-escuela-comunidad", en Revista Interuniversitaria de Formación del Profesorado, vol. 27, núm. 3, septiembre-diciembre, pp. 201-206.

Gregorutti, G. y Z. Charles-Marcel (2013), "Mejorando la relación entre la universidad y la comunidad: el caso de la Universidad de Montemorelos", en Apuntes Universitarios, vol. III, núm. 2, julio-noviembre, pp. 109-124.

Hernández, A. H. (2009), La investigación-acción como método. Una mirada desde la organización laboral, La Habana, Publicaciones Acuario.

Hernández-Sampieri, R., C. Fernández-Collado y P. Baptista-Lucio (2010), Metodología de la investigación, $5^{\mathrm{a}}$ edición, México, Editorial McGraw-Hill/ Interamericana Editores.

Lenin, V. I. (1976), "Materialismo y Empiriocriticismo", en Obras Escogidas en doce tomos, Tomo IV, 1908 y 1915, Moscú, Editorial Progreso.

Martín, G. (2005), Metódica y melódica de la animación cultural, Venezuela, Fundación para la Cultura y las Artes.

Martínez, Dianelkys (2017), Taller universidad y comunidad: una relación sociocultural, Cuba, Universidad de Pinar del Río "Hermanos Saíz Montes de Oca".

Melero, N. y R. Fleitas (2015), "La investigación acción participativa en procesos de desarrollo comunitario: una experiencia de cooperación interuniversitaria en el barrio de Jesús María, La Habana Vieja (Cuba)", en Pedagogía Social. Revista Interuniversitaria, núm. 26, pp. 203-228.

Mc Cowan, T. (2016), "Universities and the post-2015 development agenda: an analytical framework", en Higher Education, núm. 72, pp. 505-523.

Morin, E. (1999), Los siete saberes necesarios para la educación del futuro, Francia, UNESCO.

Pérez-Pérez, I. (2014), "Animación sociocultural, 
desarrollo comunitario versus educación para el desarrollo: una experiencia integradora en educación superior", en Revista Iberoamericana de Educación Superior, vol. V, núm. 12, pp. 157-172.

PNUD (2004), "Informe sobre desarrollo humano. La libertad cultural en el mundo diverso de hoy", <http:/ / hdr.undp.org/sites/default/files/hdr_2004_es.pdf> [Consulta: septiembre de 2015].

Ruíz, L. (2011), "Impacto de la Ley del Servicio Comunitario del Estudiante de Educación Superior en el Núcleo Universitario "Rafael Rangel"-Trujillo", en Educere, vol. 15, núm. 50, pp. 251-264.

Sánchez, J. e Y. Caldera (2013), "Planificación y participación comunitaria en el contexto universitario", en Investigación y Postgrado, vol. 28, núm. 2, juliodiciembre, pp. 61-79.

ONU (2015), "Transformar nuestro mundo: la Agenda 2030 para el Desarrollo Sostenible", <http://onu. org.cu/files/files/Transformar_nuestro_mundo_la_ Agenda_2030_para_el_Desarrollo_Sostenible.pdf> [Consulta: diciembre de 2016].

UNESCO (2015), "Documento de posición sobre la educación después de 2015", <http://unesdoc.unesco. org/images/0022/002273/227336s.pdf> [Consulta: diciembre de 2016].

UNESCO (2014a), "Reunión Mundial sobre la Educación para Todos", <http://www.unesco.org/new/fileadmin/ MULTIMEDIA/FIELD/Santiago/pdf/MuscatAgreement-ESP.pdf> [Consulta: diciembre de 2016].

UNESCO (2014b), "Conferencia Mundial de la UNESCO sobre la Educación para el Desarrollo Sostenible", <http://unesdoc.unesco.org/images/0023/002328/ 232888S.pdf $>$ [Consulta: diciembre de 2016].
UNESCO (2012), "Educación para el Desarrollo Sostenible. Libro de consulta", <http://unesdoc. unesco.org/images/0021/002167/216756s.pdf> [Consulta: diciembre de 2016].

UNESCO (2010), "Cultura y desarrollo. Evolución y perspectivas", <http://www.unescoetxea.org/ dokumentuak/Cultura_desarrollo.pdf $>$, [Consulta: enero de 2017].

UNESCO (2009), "Conferencia mundial de educación superior 2009", <http://www.unesco.org/education/ WGHE2009/comunicado_es.pdf> [Consulta: diciembre de 2016].

UNESGO (2006), "Directrices de la UNESCO sobre la educación intercultural", <http://eib.sep.gob.mx/ CGEIB/wp-content/uploads/2015/06/147878s. pdf> [Consulta: enero de 2017].

UNESCO (2005), "Convención sobre la protección y promoción de la diversidad de las expresiones culturales", <http://unesdoc.unesco.org / images/0014/001429/142919s.pdf> [Consulta: junio de 2015].

UNESCO (1998), "Declaración Mundial sobre la Educación Superior en el siglo XXI: Visión y Acción", <http:// unesdoc.unesco.org/images/0011/001163/116345s. pdf $>$ [Consulta: diciembre de 2016].

UNESCO (1994), Cultura y Desarrollo. Estudio, <http:// unesdoc.unesco.org/images/0009/000970/097070s. pdf> [Consulta: junio de 2015].

UNESCO (1982), "Conferencia Mundial sobre las Políticas Culturales", <http://unesdoc.unesco.org/ images/0005/000525/052505sb.pdf> [Consulta: junio de 2015]. 